\title{
Temperature Dependence of Radiation Induced Conductivity in Insulators
}

\author{
JR Dennison $^{\mathrm{a}^{*}}$, Jodie Gillespie ${ }^{\mathrm{a}}$, Joshua Hodges ${ }^{\mathrm{a}}$, RC Hoffmann ${ }^{\mathrm{a}}$, \\ J Abbott $^{\mathrm{a}}$, Steven Hart ${ }^{\mathrm{a}}$ and Alan W. Hunt ${ }^{\mathrm{b}}$ \\ ${ }^{a}$ Physics Department, Utah State University 4415 Old Main Hill, Logan, UT 84322, USA \\ ${ }^{b}$ Idaho Accelerator Center, Idaho State University, Pocatello, ID 83209, USA
}

\begin{abstract}
This study measures Radiation Induced Conductivity (RIC) of Low Density Polyethylene (LDPE) over temperatures ranging from $\sim 110 \mathrm{~K}$ to $\sim 350 \mathrm{~K}$. RIC occurs when incident ionizing radiation deposits energy and excites electrons into the conduction band of insulators. Conductivity was measured when a voltage was applied across vacuum-baked, thin film LDPE polymer samples in a parallel plate geometry. RIC was calculated as the difference in sample conductivity under no incident radiation and under an incident $\sim 4 \mathrm{MeV}$ electron beam at low incident fluxes of $10^{-4}-10^{-1} \mathrm{Gr} / \mathrm{sec}$. The steady-state RIC was found to agree well with the standard power law relation, $\sigma_{\text {RIC }}=k_{R I C} \cdot \dot{D}$

between conductivity, $\sigma$ and adsorbed dose rate, $\dot{D}$. Both the proportionality constant, $k_{R I C}$, and the power, $\Delta$, were found to be temperature dependant above $\sim 250 \mathrm{~K}$, with behavior consistent with photoconductivity models developed for localized trap states in disordered semiconductors. Below $250 \mathrm{~K}$, kRIC and $\Delta$ exhibited little change. The observed difference in temperature dependence might be related to a structural phase transition seen at $T_{\beta} \sim 256 \mathrm{~K}$ in prior studies of mechanical and thermodynamic properties of LDPE.
\end{abstract}

Keywords: radiation induced conductivity; electron transport, radiation effects, polymers, dielectrics PACS: 61.80.Az; 61.80.Fe; 61.82.Ms; 72.80.Le; 73.61.Ph; 77.22.Jp

\section{INTRODUCTION}

We report on measurements of Radiation Induced Conductivity (RIC) of thin film Low Density Polyethylene (LDPE) samples. RIC occurs when incident ionizing radiation deposits energy in a material and excites electrons into conduction states. RIC is calculated as the difference in sample conductivity under an incident flux and "dark current" conductivity under no incident radiation.

The primary focus of this study is the temperature dependence of the steady state RIC over a wide range of absorbed dose rates, from cryogenic temperatures to well above room temperature. The measured RIC values are compared to theoretical predictions of dose rate and temperature dependence based on photoconductivity models developed for localized trap states in disordered semiconductors. We also investigated the variation of RIC as a function of

\footnotetext{
* Corresponding author. Tel.: +1 435797 2936, fax: +1 435797 2492, E-mail address: JR.Dennison@usu.edu.
}

material, applied electric field, and incident beam energy parameters.

\section{THEORY}

Conductivity, $\sigma$, is a measure of the transport of charged particles under the influence of an applied electric field within a material. Theoretical models of conductivity in highly insulating materials, such as the polymers or ceramics, are most often based on hopping conductivity models involving localized trapped states. The key information to characterize a given material in such models is the number, occupation and distribution in energy, $n(E ; T)$, of the localized states found within the band gap between the top of the valence band and the bottom of the conduction band. These models were most often developed for disordered semiconducting materials, and have been shown to be quite effective in describing electron transport in these types of semiconductors. ${ }^{1}$ However, for highly insulating materials-and polymers in particular-the applicability and the validity of the assumptions 
inherent in these models are unclear. For example, trapping sites in highly disordered polymeric materials are not uniform and evenly spaced and have higher densities than in semiconductors. The limited experimental evidence to date suggests that the hopping conductivity models do, in fact, describe some basic features of polymers. ${ }^{2}$

RIC is the enhancement in conductivity of a material due to deposition of energy by incident high energy radiation. As insulators are bombarded with a flux of high energy radiation, the large energy of the incident particles is shared with many bound (valence) electrons within the material that are excited into higher energy levels in the conduction band, in a manner analogous to the effects of thermal energy on dark current conductivity. The conductivity of the material is therefore enhanced by the absorbed energy per unit mass (dose, $D$ ), rather than by direct charge deposition from the incident radiation. This is illustrated by various studies of RIC versus radiation dose rate, $\dot{D} .^{3}$

Standard theories of RIC predict that $\sigma_{R I C}$ is proportional to $\dot{D}$ raised to the power $\Delta$,

$$
\sigma_{R I C}(\dot{D}, T)=k_{R I C}(T) \cdot \dot{D}^{\Delta(T)}
$$

with proportionality constant, $k^{2,4,5}$ Both $k$ and $\Delta$ are material dependent parameters, that can in general depend on $T$. $k$ for most organic dielectrics are typically up to two or more orders of magnitude smaller than inorganic dielectrics. ${ }^{2} \Delta$ usually lies between 0.5 and 1.0, with higher values being more common. As with hopping conductivity models, we expect that $\sigma_{R I C}$ will be proportional to the number of charge carriers. ${ }^{2,5}$ At higher fluxes and incident energies, the radiation can produce new traps via radiation damage, leading to enhanced conductivity; ${ }^{6}$ such dependence typically occurs at $10^{4} \mathrm{Gr}$ or more for polymers ${ }^{3}$ and so will not be considered here.

The theory of thermal assisted hoping conductivity provides a model for the temperature, $T$, and electric field, $E$, dependence of the dark current conductivity of materials. ${ }^{7}$ This basic theory has been extended to model excitation of electrons from the valence band into the conduction band by high energy radiation and their subsequent decay into a distribution of localized trapped states with energies near the bottom of the conduction band (see Fig. 1). A theory of steady state photoconductivity in disordered semiconductors was developed by Rose that predicts the $T$ and $\dot{D}$ dependence of $k$ and $\Delta .{ }^{8}$ Fowler adapted this to model RIC. ${ }^{7,9}$ As with Mott theory for thermally assisted hopping conductivity, Rose and Fowler assume that only electron conduction is considered (e.g., holes are assumed immobile), that electrons in the extended

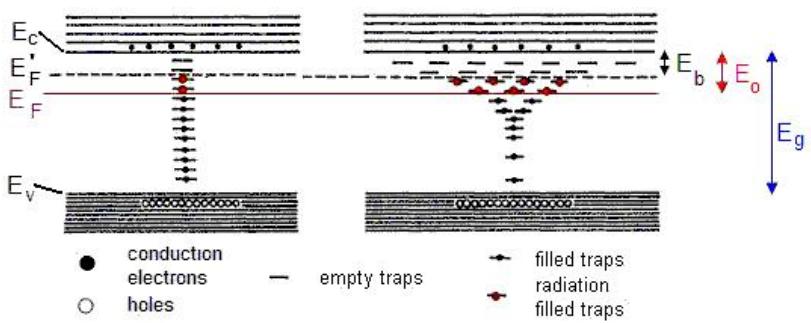

FIGURE 1. Model of conduction in disordered materials showing (Left) uniform and (Right) exponential energy distribution of localized trap states. Energies noted are: $E_{C}$, bottom of conduction band; $E_{F}$, steady-state Fermi level due to irradiation; $E_{F}$, dark current Fermi level; $E_{V}$, top of valence band; $E_{b} \equiv E_{C^{-}} E_{F} ; E_{o} \equiv E_{C^{-}} E_{F}$; and $E_{g} \equiv E_{C^{-}} E_{V}$.

states of the conduction band act as nearly free electrons, and that space charge is negligible (e.g., only bulk effects are considered and the bulk is charge neutral). RIC is predicted to depend on the energy distribution of the trapped states within the conduction band and the occupancy of these states, as well as the mean lifetimes of the photocarriers in the conduction band and the electrons in the trapped states. By arguing that in equilibrium: (i) the rate of carriers excited by the radiation from the valence band into the conduction band must equal the rate of recombination of these photoelectrons with stationary holes (ii) that high energy radiation acts to completely fill additional trapped states up to the steady-state Fermi level due to irradiation, $E_{F}$, , (iii) the number of conduction electrons excited by the high energy radiation per unit volume and time $f=\dot{D} \rho_{m} / \varepsilon$, and (iv) the distribution of trapped states exponentially decreased below the conduction band edge as $n_{b}(E)=n_{o} \exp \left(-E_{b} / k_{B} T_{1}\right)$ at a rate scaled by $T_{1}$ equal to the temperature at which traps were "frozen in" as the material cooled, Rose showed that

$$
\Delta(T)=\frac{T_{1}}{T} / T+T_{1}
$$

and

$$
k_{R I C}(T)=e \mu_{o}\left[\left(\frac{\rho_{m}}{s \Sigma n_{o} T_{1}}\right)\left(\frac{m_{e}^{*}}{3 k_{B} T}\right)^{\frac{1}{2}}\right]^{\Delta(T)}\left[2\left(\frac{\sqrt{m_{e}^{*} m_{h}^{*}} k_{B} T}{2 \pi \hbar^{2}}\right)^{3 / 2}\right]^{1-\Delta(T)}
$$

(3)

Here, $s$ is the capture cross section of conduction electrons by fixed holes, $\Sigma$ is the average energy absorbed to excite an electron from the valence band into the conduction band; $m_{e}{ }^{*}$, and $m_{h}{ }^{*}$ are the electron and hole effective masses; and $\rho_{m}$ is the mass density. The electron mobility $\mu_{o}$ is typically assumed to be independent of $T ., 2,5,8,11$ 


\section{EXPERIMENT}

Samples studied were branched low density polyethylene (LDPE) (Goodfellow, ASTM type I) of $(125 \pm 0.4) \mu \mathrm{m}$ thickness with a density of $0.92 \mathrm{~g} / \mathrm{cm}^{3}{ }^{11}$ an estimated crystallinity of $50 \%,{ }^{2}$ and a relative dielectric constant of 2.26. ${ }^{11}$ All samples were preconditioned for testing using appropriate parts of ASTM 618. ${ }^{12}$ They were chemically cleaned with spectral grade methanol prior to a bakeout at $338( \pm 1)$ $\mathrm{K}$ (well below the LDPE structural transition temperature at $\sim 90{ }^{\circ} \mathrm{C}$ ) under $\sim 10^{-3} \mathrm{~Pa}$ vacuum for $>68$ $\mathrm{hr}$ to eliminate absorbed water and volatile contaminants; samples conditioned in this manner had a measured outgassing rate of $<0.05 \%$ mass loss/day at the end of bakeout as determined with a modified ASTM 495 test procedure. ${ }^{13}$ After conditioning, the samples and sample window assembly were stored in an inert dry nitrogen environment to minimize water absorption before being mounted on the RIC chamber for testing. This low-humidity, vacuum environment reduced adsorbed and absorbed water. During vacuum bakeout, the samples were adjacent to grounded surfaces to largely dissipate residual charge in the thin film materials via the thermally enhanced dark current conductivity. Electrostatic breakdown field strength of conditioned samples was measured in a separate test chamber to be $2.9( \pm 0.3) 10^{8} \mathrm{~V} / \mathrm{m}$, using a modified ASTM D 3755 test procedure ${ }^{14}$ at room temperature under $<10^{-2} \mathrm{~Pa}$ vacuum with a voltage ramp rate of 20 $\mathrm{V}$ steps each sec. Sample conditioning can affect conductivity by up to 1 to 3 orders of magnitude. ${ }^{15}$

Dark current conductivity as a function of temperature and electric field for conditioned samples was measured without incident radiation in a separate constant voltage resistivity test chamber maintained at $\sim 10^{-3} \mathrm{~Pa}^{16}$ The measurements were made with a modified ASTM D 257 test procedure ${ }^{17}$ using a standard thin film capacitor configuration (Fig. 2g) with very good electrical shielding and low-noise cabling using guarded highly-polished OFHC $\mathrm{Cu}$ electrodes with an area of $2.0 \mathrm{~cm}^{2}$ and $\sim 0.2 \mathrm{MPa}$ clamping force per unit area. Samples were cooled to $\sim 100 \mathrm{~K}$ through contact with a $\ell-\mathrm{N}_{2}$ reservoir and then allowed to warm up at an average rate of $\sim 10 \mathrm{~K} / \mathrm{hr}$. Additional measurements were made by heating the sample to $\sim 340 \mathrm{~K}$ and then letting the sample cool to room temperature at comparable cooling rates. The conductivity showed two distinct regions above and below a critical temperature at $T_{c r}=268 \pm 2 \mathrm{~K}$, with an abrupt increase in the slope of the conductivity at higher temperatures. ${ }^{18}$

Radiation induced conductivity was studied in a custom test chamber with a modified thin film capacitor configuration (Fig. 2g) similar to those used
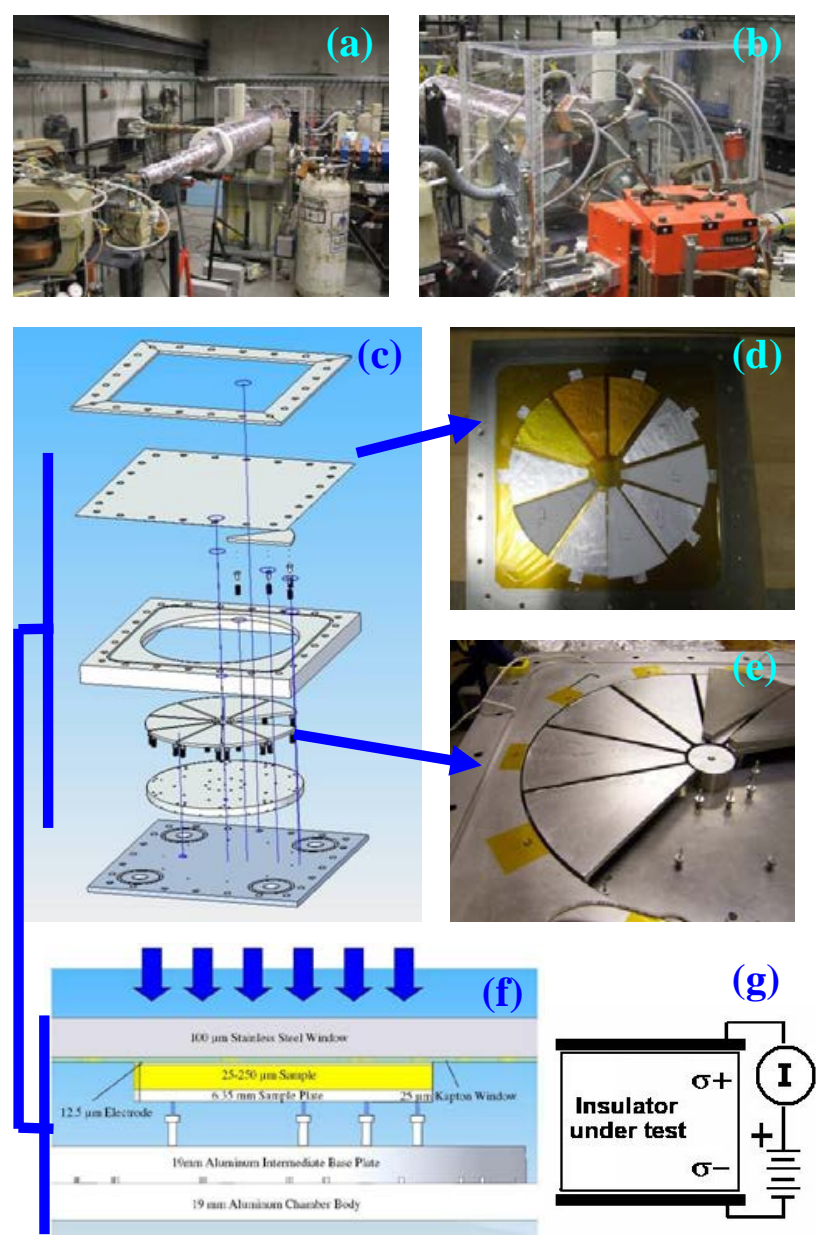

FIGURE 2. RIC vacuum chamber details. (a) IAC beam line configuration showing (left to right) accelerator, beam shutter, He-filled drift tube, cryogenic enclosure, sample window, and RIC chamber. (b) Rear view of RIC chamber showing EMI interference shielding cable conduits, vacuum line and cryogenic enclosure. (c) Exploded view of the RIC chamber. (d) Ten polymer RIC samples $\left(80.0 \mathrm{~cm}^{2}\right)$ mounted on a $100 \mu \mathrm{m}$ thick stainless steel vacuum window, shown from the vacuum chamber side. (e) RIC chamber interior showing the high voltage "pie wedge" electrodes. (f) Test configuration for RIC tests in the USU chamber. Diagram shows, from top to bottom, the vacuum window, insulation, thin conductive grounded electrode, test sample, thick high voltage "pie wedge" electrode, sapphire rod standoffs, thick grounded baseplate, and grounded chamber vacuum wall. Note the $10 \mathrm{x}$ vertical exaggeration for elements above the sapphire standoffs. Arrows show the direction of incident high energy beam. (g) Schematic of constant voltage dark current resistivity test configuration.

previously. ${ }^{5,19-21}$ The current was measured through samples sandwiched between two parallel plate electrodes with a constant applied voltage across them, while the $35 \mathrm{~cm}$ diameter sample array was subject to intense penetrating radiation. At $10^{2} \mathrm{~V}$ to 
$10^{3} \mathrm{~V}$ applied voltage, the current resolution (typically $I_{\text {res }} \approx 2 \mathrm{pA}$ ) of the picoammeter (Keithley 6486) and 10 channel multiplexing apparatus (Keithley 7002), coupled with the relatively large sample area $(A=80$ $\mathrm{cm} 2$ ), limited the measurable conductivity to $\sim 10^{-18}$ to $10^{-19}$ ohm-cm based on $\sigma_{\text {res }}=I_{\text {res }} d / V A .^{20,21}$ Note that low field or low temperature dark current conductivites for some of the samples were below this detection limit of the instrument.

Ten thin film RIC samples were cut to match the shape of the pie electrodes (see Fig. 2e), conditioned, and then mounted on the sample window assembly (Fig. 2d). Figures 2c and 2f show the cross section of the test configuration for RIC measurements: from top to bottom there were (i) the $101 \mu \mathrm{m}$ thick grounded stainless steel sheet that acted as a vacuum window and a substrate on which the samples were mounted, (ii) a $25 \mu \mathrm{m}$ Kapton $\mathrm{HN}$ insulation sheet to electrically isolate the subsequent electrode, (iii) a $12 \mu \mathrm{m} \mathrm{Al}$ foil conductive grounded electrode made as thin as practical to minimize the charge deposited in the electrode foil by the high energy beam (The area of the electrodes-excluding the tabs visible in Fig. 2d used to make electrical connection to the electrometer circuit—determined A.), (iv) the test sample, (v) a 6.35 $\mathrm{mm}$ thick high voltage "pie wedge" electrode designed to stop the incident high energy radiation and to support the pressure load on the vacuum window, (vi) four sapphire rod standoffs to support each "pie wedge" and provide a very high leakage path to ground for the high voltage electrodes, (vii) a $19 \mathrm{~mm}$ thick grounded baseplate, and (viii) a grounded chamber vacuum wall. Vacuum pressure on the window provided a $10^{5} \mathrm{~Pa}$ force per unit area to assure good electrical contact.

Figure 2b shows a rear view of RIC chamber with EMI interference shielding cable conduits, vacuum line and cryogenic enclosure. The metal vacuum chamber, RF cable shielding, and tight EMI enclosure for all test electronics reduced interference in all low current measurements in the noisy accelerator hall environment below instrumental resolution. Temperatures as low as $\sim 100 \mathrm{~K}$ were achieved using a $\ell-\mathrm{N}_{2}$ reservoir, while resistive heaters were used to reach high temperatures up to $\sim 340 \mathrm{~K}$. Because of the need to achieve uniform temperatures of multiple large area samples in contact with the thin vacuum window while under vacuum, the entire chamber was cooled, necessitating a large plexiglass enclosure around the sample (Fig. 2b) for thermal insulation and to avoid condensation on the window. The chamber was held at constant temperature for $>90$ min under constant applied electric field to reach dark current equilibrium at the initial $\mathrm{T}$ before RIC measurements began. Sample temperature was monitored to $\pm 2 \mathrm{~K}$ with
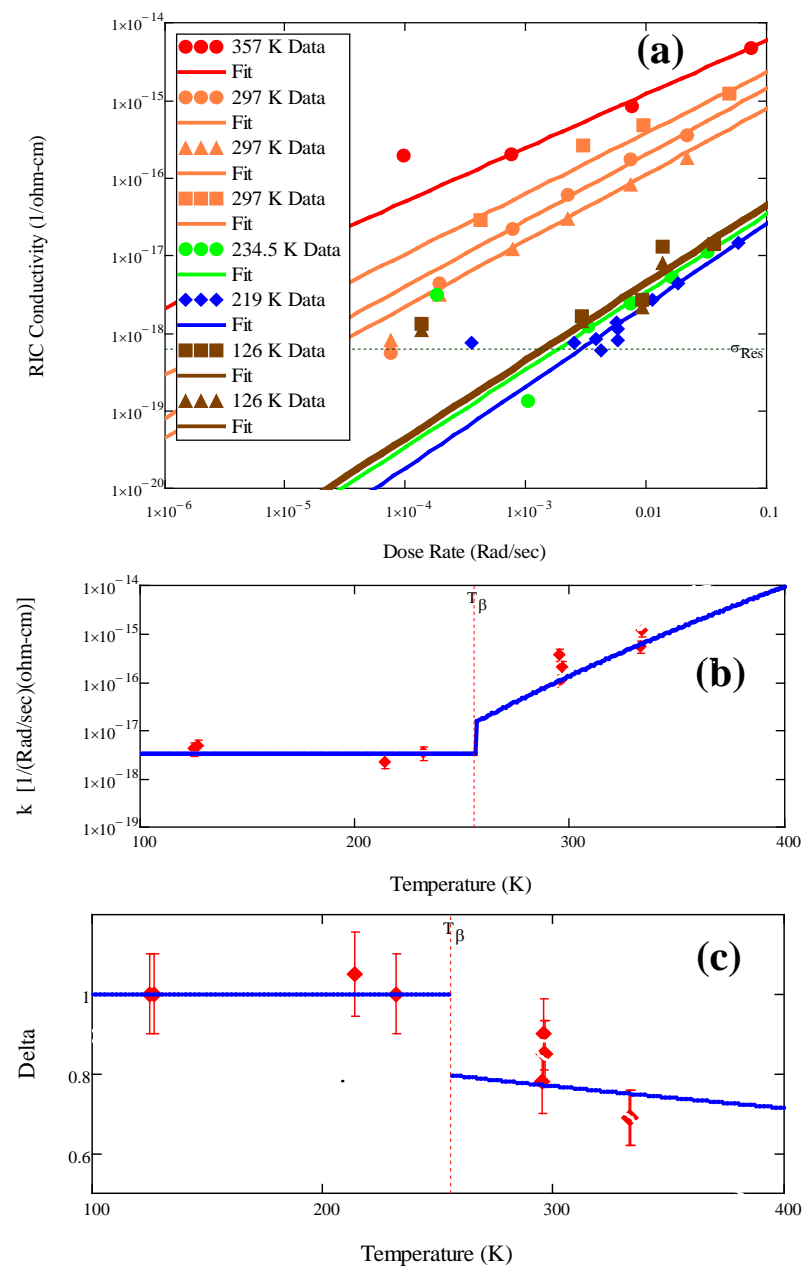

FIGURE 3. Radiation induced conductivity for $125 \mu \mathrm{m}$ thick LDPE samples with $\sim 4 \mathrm{MeV}$ electron beam incident radiation. (a) Family of curves of $\rho$ RIC vs absorbed dose rate at various temperatures listed in the legend. Lines are power law fits based on Eq. 1. A horizontal marker shows the approximate minimum measurable conductivity. (b) Temperature dependence of RIC coefficient, kRIC. Line is fit based on Eq. 3 with ko= 1.5·10-16 $(\Omega-\mathrm{cm}-\mathrm{rad} / \mathrm{s})-1$ and T1 $=500 \mathrm{~K}$. (c) Temperature dependence of RIC power law coefficient, $\Delta$. Line is fit based on Eq. 2.

several Type $\mathrm{K}$ thermocouples attached to various parts of the apparatus including the vacuum window and pie wedge electrodes.

RIC measurements were made at the Idaho Accelerator Center (IAC), using the IAC $2 \mathrm{MeV}$ to 25 $\mathrm{MeV}$ high-repetition rate pulsed-electron linear accelerator (Fig. 2a). The $5 \mathrm{MeV}$ beam used for all experiments reported here had a typical pulse width of $200 \mathrm{~ns}$ and a repetition rate of 10 to $100 \mathrm{~Hz}$; the energy distribution was moderated to $\sim 4 \mathrm{MeV}$ peak energy and broadened somewhat by $\mathrm{Al}$ scattering foils and the chamber window used to produce the large- 
area uniform-flux beam. A $\sim 5 \mathrm{~m}$ long He-filled flight tube was placed between the beam shutter assembly and scattering foils and the RIC chamber window (see Fig. 2a). Our limited measurements verified previous results that $\sigma_{\text {RIC }}$ was very largely independent of incident beam energy parameters (beam energy, pulse width, amplitude and duty cycle) ${ }^{3,20}$ and electric field strength up to $>10^{6} \mathrm{~V} / \mathrm{m}^{15}$

Tests were conducted over three orders of magnitude of dose rate from $10^{-4} \mathrm{~Gy} / \mathrm{sec}$ to $10^{-1}$ Gy/sec. The general range of incident radiation at the accelerator was set through a combination of increased source to sample separation and shielding. The specific sample dose rates were set primarily by adjusting the beam current and-at the higher dose rates-by increasing the repetition rate. Relative dose rate was monitored during RIC tests with a real-time miniature ionization chamber monitor positioned outside the RIC chamber window. Measurements showed that the incident beam was quite uniform over the full sample area, with a gaussian profile with a relative deviation from mean of $+/-5 \%$. The beam profile was confirmed and absolute dose rate calibration measurements were performed using Optically Stimulated Luminescence dosimeters (Landauer, InLight microStarReader) placed at up to 15 locations on the RIC chamber window; the sensors and reader were calibrated at the Radiological and Environmental Science Laboratory using a NIST traceable ${ }^{137}$ Cs source.

\section{RESULTS AND DISCUSSION}

Measurements were made of the RIC current (sample current minus the dark current contribution) as a function of time at constant voltage and various fixed temperatures. The steady-state RIC at each dose rate were calculated using Eq. (1) from the average equilibrium current in the plateaus of the current versus time curves, and are plotted in Fig. 3a. Data points on the graph below $\sigma_{\text {res }}$ are not reliable, as they are calculated as the small difference between two values near the current resolution limit. Power law fits based on Eq. 1 model the data well (see Fig 3a), with estimated uncertainties in the parameters $k_{R I C}$ and $\Delta$ of $\pm 20 \%$ and $\pm 10 \%$, respectively.

Both $k_{R I C}$ and $\Delta$ were found to be temperature dependant, as seen in Fig. 3. Above $\sim 250 \mathrm{~K}$, this behavior is reasonably consistent with Eqs. 2 and 3 developed for photoconductivity models of localized trap states in disordered semiconductors. Below 250 $\mathrm{K}, k_{R I C}$ and $\Delta$ exhibited little change. Measured values of $\Delta$ are in the expected range of $0.5<\Delta<1.0$ and had a constant value of 1.0 below $\sim 250 \mathrm{~K}$. Fowler reported below $\sim 250 \mathrm{~K}$ a similar jump in $\Delta$ to a constant value of $0.83 .^{5}$ The temperature behavior of $\Delta$ above $\sim 250$ $\mathrm{K}$ is modeled reasonably well by Eq. 3 with $T_{1}=500 \mathrm{~K}$ and is consistent - to within experimental uncertainties-with numerous previous studies above $\sim 253 \mathrm{~K}^{5,22-25}$ The average measured value of $k_{R I C}$ at room temperature value of $(2 \pm 1) \cdot 10^{-12}\left(\mathrm{Gr} \mathrm{sec}^{-1} \Omega \mathrm{m}^{-1}\right.$ is in reasonable agreement with the range of (3 to $6) \cdot 10^{-12}\left(\mathrm{Gr} \mathrm{sec}^{-1} \Omega \mathrm{m}\right)^{-1}$ from previous studies. ${ }^{5,24,25}$ The temperature behavior of $k_{\text {RIC }}$ shown in Fig. $3 \mathrm{~b}$ is in good agreement with previous temperature studies extending over a range of $\sim 120 \mathrm{~K}$ to $355 \mathrm{~K},{ }^{5,20,22}$ when these studies are normalized to the same $k_{R I C}$ at room temperature to account for modest differences in materials and methods. ${ }^{15}$

The observed abrupt changes in temperature dependence for RIC at $\sim 253 \mathrm{~K}$ and in dark current conductivity at $T_{c r}=268 \pm 2 \mathrm{~K}{ }^{18}$ may well be related to a LDPE structural phase transition seen at 250 $\mathrm{K}<T_{\beta}<262 \mathrm{~K}$ in prior studies of mechanical and thermodynamic properties. The $\beta$ transition is a structural phase transition routinely observed in branched PE, which has been associated with conformational changes along polymer chains in the interfacial matrix of disordered polymer between nanocrystalline regions in the bulk. ${ }^{26}$

\section{ACKNOWLEDGMENTS}

We acknowledge used suggestions by Alec Sim. This work was supported by a project for the James Web Space Telescope Electrical Systems Group through the NASA Goddard Space Flight Center.

\section{REFERENCES}

1. R. Zallen,, The Physics of Amorphous Solids, New York: Wiley, 1983, p. 252.

2. H.J. Wintle, "Conduction Processes in Polymers," in Engineering Dielectrics - Volume IIA: Electrical Properties of Solid Insulating Materials: Molecular Structure and Electrical Behavior, American Society for Testing and Materials, R. Bartnikas, Editors, Philadelphia: Am. Soc. for Testing and Materials, 1983.

3. F.J. Campbell "Radiation Effects of the Electrical Properties of Solid Insulation," in Engineering Dielectrics - Volume IIA: Electrical Properties of Solid Insulating Materials: Molecular Structure and Electrical Behavior, American Society for Testing and Materials, R. Bartnikas, Editors, Philadelphia: Am. Soc. for Testing and Materials, 1983.

4. L. Weaver, J. Kenneth Shultis, and R. E. Faw, J. Appl. Phys. 48, 2762 (1977).

5. J. F. Fowler, Proc. Royal Soc. London A, 23, 464 (1956).

6. A. Chapiro, Atomic Radiation and Polymers, New York: Pergamon, (1960) p. 171. 
7. N.F. Mott and E.A. Davis, Electronic Processes in NonCrystalline Materials, $2^{\text {nd }}$ Ed., Oxford: Oxford University Press, (1979).

8. A. Rose, RCA Rev., 12, 362 (1951).

9. J.F. Fowler, "X-ray Induced Conductivity in Insulating Materials,” Ph.D. Thesis, Un. London, 1955.

10 A.P. Tyutnev, V.S. Saenko, E.D. Pozhidaev and V. A. Kolesnikov, High Performance Polymers, 17 (2005) 175-192..

11. "Material Information - Polyethylene Low Density LDPE,” Devon, PA: Goodfellow, January 20, 2006.

12. ASTM D-618-00, "Standard Practice for Conditioning Plastics for Testing," West Conshohocken, PA: American Society for Testing and Materials, 2001.

13. ASTM D 495-99, "Standard Test Method for HighVoltage, Low-Current, Dry Arc Resistance of Solid Electrical Insulation," West Conshohocken, PA: American Society for Testing and Materials, 2001.

14. ASTM D 3755, "Standard Test Method for Dielectric Breakdown Voltage and Dielectric Strength of Solid Electrical Insulating Materials under Direct-Voltage Stress," West Conshohocken, PA: American Society for Testing and Materials, 2001.

15. V. Adamec and J. H. Calderwood, J. Phys. D: Appl. Phys., 14, 1487 (1981).

16. J.R. Dennison, Prasanna Swaminathan, Randy Jost, Jerilyn Brunson, Nelson Green, and A. Robb Frederickson, IEEE Trans. Plasma Sci., 34, 2191 (2006).

17. ASTM D 257-99, "Standard Test Methods for DC Resistance or Conductance of Insulating Materials” West Conshohocken, PA: American Society for Testing and Materials, 2001.

18. J.R. Dennison and J. Brunson, IEEE Trans. Plasma Sci. 36 (2008).

19. A.R. Frederickson, IEEE-Trans. Nucl. Sci., NS-24(6), 2532 (1977).

20. R.A. Meyer. F.L. Bouquet and R.S. Alger, J. Appl. Phys. 27(9) (1956) 1012.

21. L. Levy, D. Sarrail, and J.M. Siguier, Third European Symposium on Spacecraft Materials In Space Environment, 1985, p. 123.

22. K. Yahagi and A. Danno, J. Appl. Phys., 34, 804 (1963).

23. S. Mayburg and W.L. Lawrence, J. Appl. Phys. 23, 1006 (1956).

24. J.H. Coleman and D. Bohm, J. Appl. Phys. 24, 497 (1953).

25. C.L. Hanks and D.J. Hammond, REIC Report No. 46, Columbus, OH: Radiation Effects Center, 1969. p. 39.

26. R. Popli, M. Glotin, L. Mandelkern, and R.S. Benson, J. Polymer Sci. Polymer Phys. Ed, 22, 407 (1984). 